“ (C) 2016 IEEE. Personal use of this material is permitted. Permission from IEEE must be obtained for all other uses, in any current or future media, including

reprinting/republishing this material for advertising or promotional purposes, creating new collective works, for resale or redistribution to servers or lists, or reuse of any copyrighted component of this work in other works." 


\title{
Spatial Prediction in Mobile Robotic Wireless Sensor Networks with Network Constraints
}

\author{
Ravindra Ranasinghe ${ }^{1}$ and Sarath Kodagoda ${ }^{1}$
}

\begin{abstract}
In recent years mobile robotic wireless sensor networks have been a popular choice for modelling spatial phenomena. This research is highly demanding and non-trivial due to challenges from both network and robotic aspects. In this paper, we address the spatial modelling of a physical phenomena by introducing the network connectivity constraints while the mobile robots are striving to achieve the minimum modelling mismatch in terms of root mean square error (RMSE). We have resolved it through Gauss markov random field based approach which is a computationally efficient implementation of Gaussian processes. In this strategy, the mobile robotic wireless sensor nodes (MRWSN) are centrally controlled to maintain the connectivity while minimizing the RMSE. Once the number of MRWSN reach their maximum coverage, a new MRWSN is requested at the most informative location. The experimental results are convincing and they show the effectiveness of the algorithm.
\end{abstract}

\section{INTRODUCTION}

The deployment of wireless sensor networks have significant impact on both scientific adventures and our daily life. With advances in sensing, wireless communication (low power RF) and computing, in recent years, Wireless Sensor Networks (WSN) have become a viable and very effective means for data intensive observation in various application domains [1], [2]. Often, these WSN are required to be deployed in areas without infrastructure support, in hostile fields, and harsh environments. Applications include spatially $\&$ temporally dense environmental monitoring, battle field monitoring seismic structure response study, precision farming, traffic monitoring, disaster prone areas, and hazardous zones [3]-[7]. In large scale deployment of WSN, coverage and connectivity are basic requirements to capture the environmental phenomena and to report information to the sink (the base station).

In order to accomplish this goal, researchers have recognised the Mobile Robotics Wireless Sensor Networks, a special branch in WSN where the mobility of the sensor nodes has been exploited for improving communication coverage and sensing [8]. These Mobile Robotics Wireless Sensor Networks are flexible so that they can adopt to changes in the environment and to enhance efficiency of data collection. Moreover in the event of sensor node failures, remaining nodes can reorganize to continue the data collection efficiently.

\footnotetext{
*This work was supported by the Centre for Autonomous Systems, University of Technology Sydney

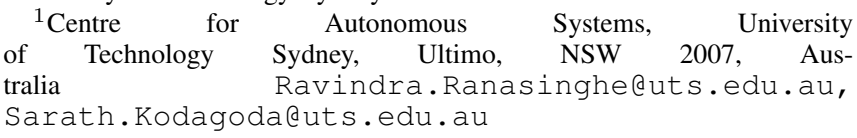

Typically, these wireless sensor networks are hybrid networks with both static nodes and mobile nodes (i.e. Mobile Robotics Wireless Sensor Nodes (MRWNs)). Availability of MRWSNs enables to develop efficient sensor node deployment strategies dynamically (i.e. sampling paths of the MRWSNs) to collaboratively move in a space to effectively monitoring the physical field.

In literature, numerous schemes have been proposed for the deployment of MRWNs in large candidate regions addressing both the coverage and the connectivity. In [9] a potential field based method was proposed to uniformly distribute the sensor nodes in a region with physical obstacles. In [10][35] a cluster based virtual force method was proposed for randomly spread MRWNs to form clusters based on their physical locations. Nodes may exert either attractive or repulsive forces depending on the relative distance between them to determine the distribution of the nodes while maintaining the connectivity. The Connectivity Preserved Virtual Force Method (CPVF) proposed in [11] logically divides the region into several floor lines separated by a distance $d=2 r_{s}$ where $r_{s}$ is the sensing range of sensor nodes. Initially, MRWNs are randomly dispersed and after that they start moving towards their nearest floor line. Then they start moving along the floor lines until they find a connectivity to the sink. The Voroni Based Algorithm [12] works on the pull driven principal where MRWNs attempts to cover the holes in respective Voroni polygons. Motivated from the balance of molecules within certain compound in terms of the density, the Distributed Self Spreading Algorithm (DSSA) presented in [13] attempts to uniformly distribute the sensor nodes by repeatedly locating them in the region to achieve a desired density. In [12] authors have presented a Vector Based Algorithm to uniformly distribute nodes in the region. The coverage over the region is accomplished by means of pushing forces between sensor nodes that resembles the repulsive force that exists between similar charged particles.

We argue that deploying a large number of sensors uniformly in the candidate region while maintaining the connectivity may not be the most optimal approach for collecting measurements in the spatial field. We would rather like to move MRWSNs to the most informative locations while maintaining the connectivity.

In [?], authors have presented an information theoretic approach to determine an optimal number of MRWSNs and their optimal sampling path using a Gauss Markov Random Field (GMRF) to model the spatial field. In that work authors assumed that the connectivity between nodes and the sink exists and connectivity was not considered as part of the 
optimization process. In this work we focus on positioning of the MRWSNs in the application region (i.e. coverage) while maintaining the network connectivity to achieve a desired level of accuracy. In essence our aim is to move 'sufficient' number of sensor nodes to most informative locations in each iteration to capture the physical phenomena most effectively while ensuring the connectivity of each sensor node to the sink.

The remainder of this paper is organized as follows. In Section II, Gauss Markov random field based spatial prediction model is described. Robotic and network related constraints are formulated in the Section III. The adaptive sampling strategy for the MRWSN are proposed in the Section IV. Experimental results are presented in Section V and Section VI concludes the paper with future directions.

\section{SPATIAL SAMPLING WITH MOBILE SENSOR WIRELESS SENSOR NETWORKS}

\section{A. Gauss Markov Random Fields (GMRF)}

The Gauss Markov Random Fields provides a computationally efficient alternative to Gaussian Processes. It is a discretely indexed Gaussian field and we use the models represented in [14].

Let's consider a finite set of spatially observed locations $\mathbf{u}=\left\{u_{1}, u_{2}, \ldots, u_{n}\right\} \subset \mathcal{Q}$ where $\mathcal{Q}$ is a convex polytope in $\mathbb{R}^{d}$. We assume that $u_{i}, i=1, \ldots, n$ varies continuously through the polytope $\mathcal{Q}$ and every measurement is related to a particular location in $\mathbf{u}$. Let $y(\mathbf{u})=\left\{y\left(u_{1}\right), \ldots, y\left(u_{n}\right)\right\}$ denote a vector of measurements.

The measurement model is defined by,

$$
y(\mathbf{u})=X(\mathbf{u}) \beta+z(\mathbf{u})+\varepsilon(\mathbf{u}),
$$

where, the model is consisted of a summation of a large scale component, a random field, and an identically distributed (i.i.d.) noise. $X(\mathbf{u}) \beta$ is the expectation of $y(\mathbf{u}) . X(\mathbf{u})$ is the covariates determined at location $\mathbf{u}$ and $\beta$ represents a vector of mean parameters. In the perspective of Gaussian Process [15], the term $X(\mathbf{u}) \beta$ is referred to as the mean function. $\varepsilon(\mathbf{u})$ is a noise with a zero mean and a covariance matrix $\sigma_{\varepsilon}^{2} I_{\varepsilon}$ at locations $\mathbf{u}$, where $\sigma_{\varepsilon}^{2}$ is assumed to be known and $I_{\varepsilon}$ is the $n \times n$ identity matrix. $z($.$) represents a GMRF with$ a zero mean and a precision matrix $Q$. It is to be noted that the GMRF is Markovian leading to a sparce precision matrix, which is exploited for substantial saving in computation.

\section{B. Mobile Robotic Wireless Sensors (MRWS)}

A network of $N$ number of mobile agents indexed by $i \in\{1, \ldots, N\}$ with on-board identical sensing and communication capability are assumed. They can take measurements at regular interval of times, $t \in \mathbb{Z}_{>0}$. At time $t$, agent $i$ takes a noisy measurement at its current location $s_{i} \in \mathcal{Q}$. Let's denote all the locations of MRWS at time $t$ by $s_{t}=$ $\left(s_{t, 1}^{T}, \ldots, s_{t, N}^{T}\right)^{T}$, and the measurements made by all MRWSs at time $t$ by $y_{t}=\left(y_{t, 1}, \ldots, y_{t, N}\right)^{T}$. Let's also denote the collection of all mobile sensors' locations and the collective measurements from time 1 to $t$ by $s_{1: t}=\left(s_{1}^{T}, \ldots, s_{t}^{T}\right)^{T}$, and $y_{1: t}=\left(y_{1}, \ldots, y_{t}\right)^{T}$ respectively.

\section{Spatial Prediction Model}

As shown in [16], the authors have utilized the Finite Element method [17] to project the SPDE onto a basis representation that consists of piece-wise linear basis functions described by a triangulation of the domain of interest. The triangulation is constructed by a set of non-intersecting triangles in which the triangle initial vertices are imposed at the observed locations. Moreover, for spatial prediction, additional vertices are added to complete a large triangulation. Based on this triangulation, the spatial field is discretized into $v$ spatial sites at all $v$ vertices of the triangulation.

The full conditional distribution of $z_{t}$ given $y_{1: t}$ which is also Gaussian can be specified by

$$
z_{t} \mid y_{1: t}, \theta_{t}, \beta_{t}, \sigma_{\varepsilon}^{2}, A_{t} \sim \mathcal{N}\left(\mu_{z_{t} \mid y_{1: t}}, Q_{z_{t} \mid y_{1: t}}^{-1}\right)
$$

where

$$
\begin{aligned}
\mu_{z_{t} \mid y_{1: t}}= & \mu_{z_{t}} \\
& +Q_{z_{t} \mid y_{1: t}}^{-1} A_{t}^{T}\left(\sigma_{\varepsilon}^{2} I\right)^{-1}\left(y_{1: t}-X\left(s_{1: t}\right) \beta_{t}-A_{t} \mu_{z_{t}}\right) \\
Q_{z_{t} \mid y_{1: t}}^{-1}= & Q_{t}+A_{t}^{T}\left(\sigma_{\varepsilon}^{2} I\right)^{-1} A_{t}
\end{aligned}
$$

where, $\theta_{t}$ is the hyperparameter vector and $\beta_{t}$ is the vector of mean parameters. Note that both $\theta_{t}$ and $\beta_{t}$ are unknown in this model.

\section{Parameter Estimation}

As discussed in [14], the parameters of the Gaussian field model are learned through the log-likelihood function which has the maximum values at

$$
\widehat{\beta}_{t}=\left(\left(X\left(s_{1: t}\right)^{T} \Sigma_{t}^{-1} X\left(s_{1: t}\right)\right)^{-1} X\left(s_{1: t}\right)^{T} \Sigma_{t}^{-1} y_{1: t} .\right.
$$

It is to be noted that only the variables in the distribution (2) are $\beta_{t}$ and $\theta_{t}$. Once $\beta_{t}$ is known, $\theta_{t}$ can be estimated by utilizing the maximum likelihood approach [18]. Note that the log-likelihood maximization procedure can be used recursively and for many hyperparameters.

\section{ROBOTIC AND NETWORK CONSTRAINTS}

There are three types of constraints can be present in MRWSNs: Robot related constraints, network related constraints and infrastructure related constraints. Robot related constraints are imposed specifically due to the limited capability of the robot. For example, non-holonomic constraints, speed of operation and localization errors, etc. How to efficiently dispatch the mobile sensors to find an obstacle-avoiding shortest path is a big confront. Examples of network related constrains are related to communication range, coverage and battery life. The infrastructure based constraints are related to robots operating environments. Examples of such constraints are due to presence of obstacles and non-accessible regions. It is our futuristic goal to include all the constraints however, in this paper we will restrict our scope to robot and network related constraints. 


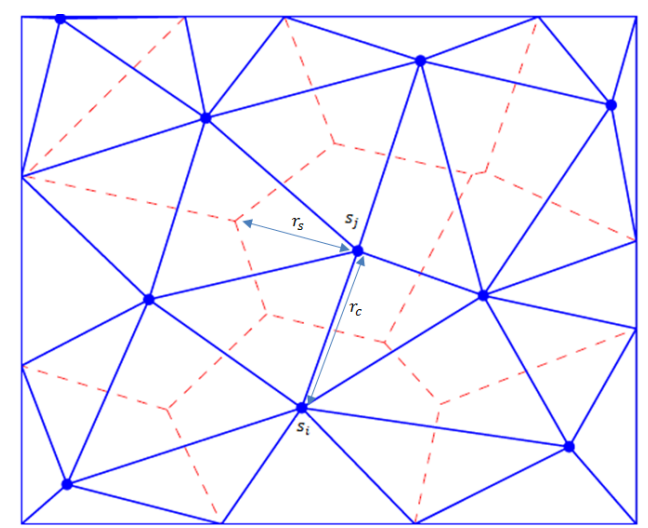

Fig. 1: The spatial filed. Communication cells are formed by red dashed lines and a mesh of a triangulation is created by blue lines.

\section{A. Robotic constraints}

The mobile robots are assumed having holonomic movements according to the discrete dynamics $s_{i}(t+1)=s_{i}(t)+$ $h_{i}(t)$, where $\left\|h_{i}\right\| \leq h_{\max } \in \mathbb{R}_{>0}$, in which $h_{\max }$ is the maximum distance an agent can move in a particular sampling interval. The robots are assumed to be capable of localizing themselves.

The sensing range $\left(r_{s}\right)$ is defined as the area in which the MRWSN is capable of taking measurements representing all targets that fall inside the area. The sensing range of a MRWSN is normally modelled as a circle in the 2D space or as a sphere in the 3D space with radius $r_{s}$ where the sensor is positioned at the center. The communication range $\left(r_{c}\right)$ of a MRWSN is centred on the sending node which is defined as the area in which the received power is sufficient to decode the signal.This is also modelled in a similar way to the sensing range. The data transmitted by a MRWSN can be received by all MRWSNs that are within the communication range $\left(r_{c}\right)$ of the transmitter. Typically, MRWSN's $\left(r_{c}\right)$ is greater than its $\left(r_{s}\right)$.

Fig.1 illustrates how the field is represented by an irregular lattice of a triangulation.

In our work, robot-robot collision avoidance is implemented based on the sensing range perspective where once a measurement is taken at the most informative location, the need for taking another measurement by another mobile robotic sensor at the same time is constrained by a radius $r_{s}$. Therefore, $\left|s_{t, i}\right|-\left|s_{t, j}\right|>r_{s}$. During a given data collection step, any point that falls inside the sensing range is forbidden for any other sensor to move in even if helps improving the RMSE (i.e. a location with higher information). In Fig.2 the circular void area centred at $(9,6)$ shows the area that is not allowed for any other sensor to move in during the current step. The algorithm has picked up the most informative connected point outside this region (represented by the green dot).

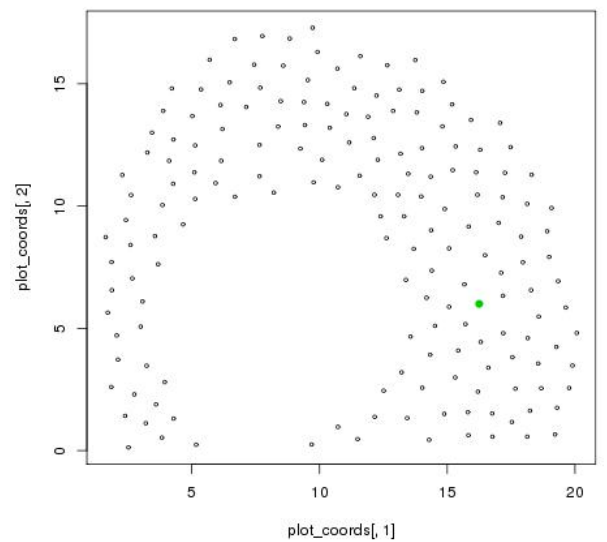

Fig. 2: Forbidden zone for placing the next sensor

\section{B. Network Constraints}

We assume a centralized communication scheme where each sensor can communicate within its range and can be routed to a common sink. Interference between the mobile sensor nodes is assumed insignificant and a Medium Access Control (MAC) protocol is assumed to address the collisions on the network. Network coverage is utmost important and each and every mobile agent is assumed to be having a communication range, $r_{c}$. If $\left|s_{t, i}\right|-\left|s_{t, j}\right|<r_{c}$ for any $i$ and $j$, where $i \neq j$, then the network is functional having atleast one path for every MRWSN to reach the sink.

\section{ADAPTIVE SAMPLING}

In this paper, an attempt was made to estimate and predict a given spatial filed using the minimum number of mobile sensory agents. This requires the robots to take optimal sampling paths that drive the MRWSN to the most informative locations to observe the physical environment while maintaining the network connectivity. It is shown that each MRWSN always looks for a next location at time instant $t+1$ given all measurements up to time $t$. In the following, we consider the one-step-ahead spatial prediction technique before the adaptive sampling approach is proposed.

\section{A. Spatial Inference through one-step-ahead Prediction}

In this section, the one-step-ahead forecast is used to predict the latent spatial field qualtities at time $t+1$, given the collective observations up to time $t$. The readers are referred to [?] for more detailed discussion. The spatial field model (1) can be rewritten as

$$
y_{1: t}=X\left(s_{1: t}\right) \beta_{t}+A_{t} z_{t}+\varepsilon\left(s_{1: t}\right),
$$

where $\varepsilon\left(s_{1: t}\right) \sim \mathcal{N}\left(\mathbf{0}, \sigma_{\varepsilon}^{2} I\right)$. Given $z_{t} \mid y_{1: t}$, the latent spatial process at time step $t+1$ can be predicted by

$$
\widehat{z}_{t+1}\left|y_{1: t}=z_{t}\right| y_{1: t}+\eta_{t+1}
$$

where $\eta_{t+1} \sim \mathcal{N}\left(\mathbf{0}, Q_{\eta, t+1}^{-1}\right), Q_{\eta, t+1}^{-1}=\sigma_{s y s}^{2} I_{\eta}, I_{\eta}$ is a $v \times v$ identity matrix, and $\sigma_{\text {sys }}^{2}$ is the system error that describes 
the changes in the elements of the parameters vector between times $t$ and $t+1$.

Now the prior conditional distribution of $\widehat{z}_{t+1}$ given $y_{1: t}$ can be obtained by

$$
\widehat{z}_{t+1} \mid y_{1: t} \sim \mathcal{N}\left(\mu_{z_{t} \mid y_{1: t}}, Q_{z_{t} \mid y_{1: t}}^{-1}+Q_{\eta, t+1}^{-1}\right) .
$$

Further, if $V T$ is a set of the vertices on the triangulation, we define a column vector $b_{t+1} \in \mathbb{R}^{v}$ at time $t+1$ as

$$
b_{t+1}(i)= \begin{cases}1, & \text { if } V T(i)=s_{t+1}(j) \text { and } j=1, \ldots, N \\ 0, & \text { otherwise. }\end{cases}
$$

Then, the posterior precision matrix of the posterior distribution of $\widehat{z}_{t+1}$ conditioned on $y_{1: t}$ and $\widehat{y}_{t+1}$ is specified by

$$
\widehat{Q}_{z_{t+1} \mid y_{1: t}, \widehat{y}_{t+1}}=\left(Q_{z_{t} \mid y_{1: t}}^{-1}+Q_{\eta, t+1}^{-1}\right)^{-1}+\frac{1}{\sigma_{\varepsilon}^{2}} b_{t+1} b_{t+1}^{T} .
$$

For the sake of simplicity, from here we will use $\widehat{Q}_{z_{t+1}}=$ $\widehat{Q}_{z_{t+1} \mid y_{1: t}, \widehat{y}_{t+1}}, Q_{z_{t}}=Q_{z_{t} \mid y_{1: t}}$, and $Q_{\eta}=Q_{\eta, t+1}$.

\section{B. Near-optimal Sampling Paths}

In recent works, the approaches mostly used to find the most informative sampling locations at the next time step $t+1$ is derived from information-theoretic criteria such as entropy [?]. These works proposed to optimize the conditional entropy that is with the closed form expressions developed on the GP models [?], [?], or on the GMRF models restricted to a regular lattice [?], [?], [19]. In this subsection, we extend the conditional entropy based adaptive sampling method on the GMRF models that are described on an irregular lattice.

The crucial idea of our proposed technique is to drive the MRWSN to the new sampling positions without disrupting the communication links so that the uncertainty at the $v$ spatial sites is minimized. Let us define the covariance matrix $\widehat{\Sigma}_{z_{t+1}}=\widehat{Q}_{z_{t+1}}^{-1}$, the conditional entropy of $\widehat{z}_{t+1} \mid y_{1: t}, \widehat{y}_{t+1}$ is computed in terms of the closed form expression (17) as

$$
H\left(\widehat{z}_{t+1} \mid y_{1: t}, \widehat{y}_{t+1}\right)=\frac{1}{2}\left\{\log \operatorname{det}\left(\widehat{\Sigma}_{z_{t+1}}\right)+v \log (2 \pi)+v\right\} \text {. }
$$

Thus, the next optimal sampling locations at time instant $t+1, s_{t+1}$, can be obtained by minimizing the uncertainty as follows

$$
s_{t+1}^{o p t}=\underset{s_{k+1}(i) \in \Omega_{V T}^{(t)}(i)}{\operatorname{argmin}} \log \operatorname{det}\left(\widehat{\Sigma}_{z_{t+1}}\right),
$$

where $\Omega_{V T}^{(t)}(i)$ is a set of the spatial sites inside $V T$ but restricted by $\Omega_{i}^{(t)}$ based on the robotic and network constraints. Since $\log \operatorname{det}\left(\widehat{\Sigma}_{z_{t+1}}\right)=-\log \operatorname{det}\left(\widehat{Q}_{z_{t+1}}\right)$, we have

$$
s_{t+1}^{o p t}=\underset{s_{k+1}(i) \in \Omega_{V T}^{(t)}(i)}{\operatorname{argmax}} \log \operatorname{det}\left(\widehat{Q}_{z_{t+1}}\right) .
$$

It can be clearly seen that this combinatorial optimization problem as proved by Ko et al. [?] is NP-hard. Usually, this issue can be efficiently addressed by employing a greedy algorithm that sequentially finds a set of the next nearoptimal sampling locations at time $t+1, s_{t+1}^{*}$ [?].

\section{Algorithm}

This section summarises the algorithm to drive MRWSNs to collect data from the filed.

At the beginning of each period, sink receives data from $N$, MRWSNs either directly or through other intermediate MRWSNs (i.e.multihop).Then the sink works out the next best location for each sensor node at time instant $t+1$ given all measurements up to time $t$ as explained in Section . Then it evaluates the RMSE based on all the readings it has received so far. If the value of the RMSE is not significantly changing between updates, it injects one or several robots to the field as it requires to gather more information from the uncovered areas.

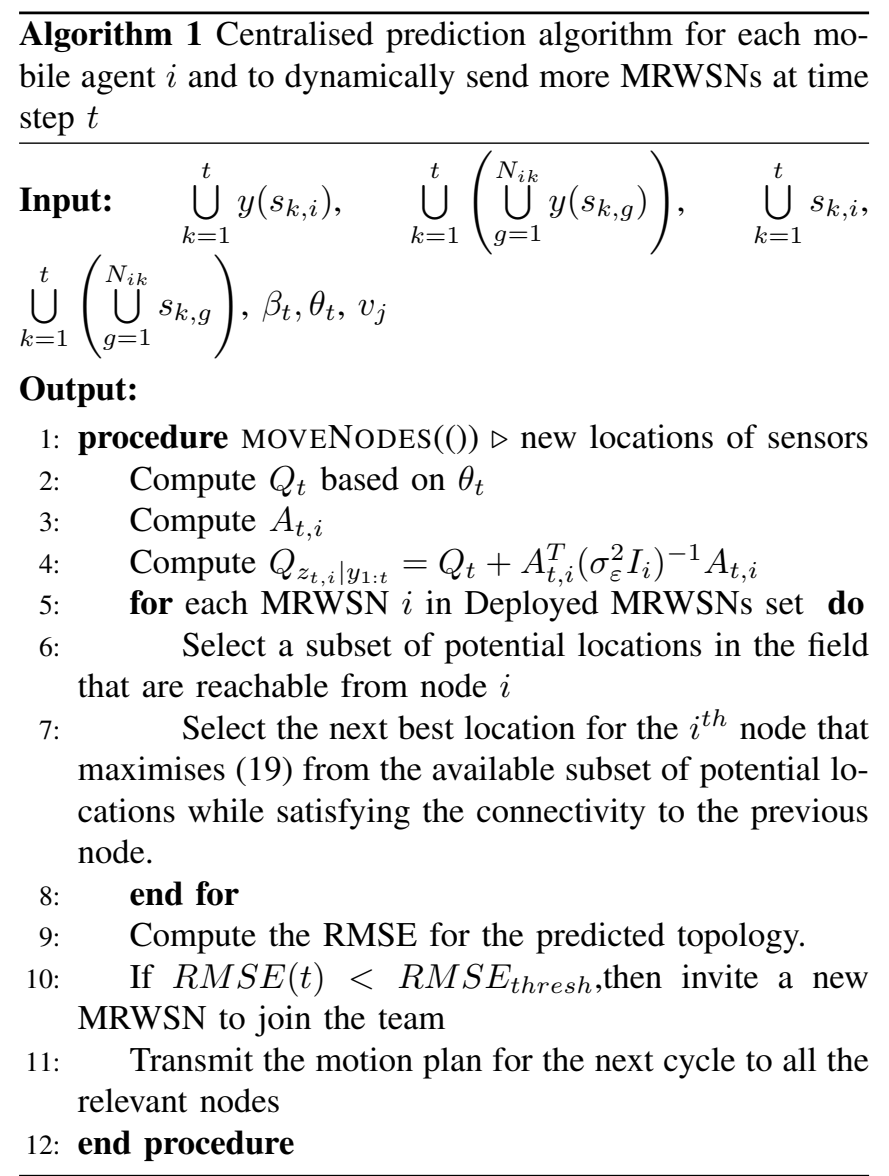

\section{EXPERIMENTAL RESUlTS}

In this section we describe experiments which try to objectively illustrates the benefits of our proposed strategy.

The numerically generated data set that was used in [19] was utilized to conduct simulation experiments. A spatial field of size 100 units 50 units was created and the physical quantity was generated within this field shown in Fig. 3a. There were $\mathrm{N}$ number of MRWSNs and each sensor node has $15 \mathrm{~m}$ communication range $\left(r_{c}\right)$ and $5 \mathrm{~m}$ sensing range $\left(r_{s}\right)$. When the first 3 MRWSNs are placed in the field they start from pre-defined locations as shown in Fig. 4a (in white dots). Locations of all the subsequent MRWSNs are algorithmically determined subjected to connectivity and RMSE constraints (Figures $4 b$ and $4 c$ ). We have assumed 


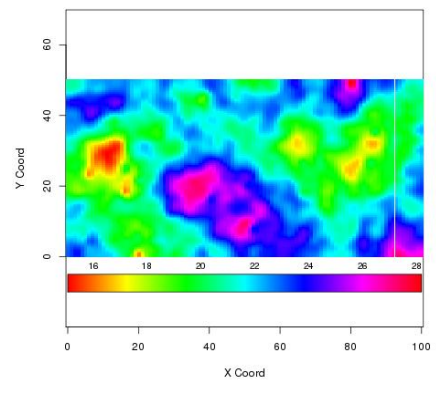

(a)

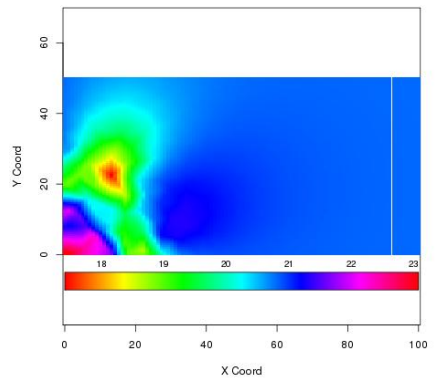

(b)

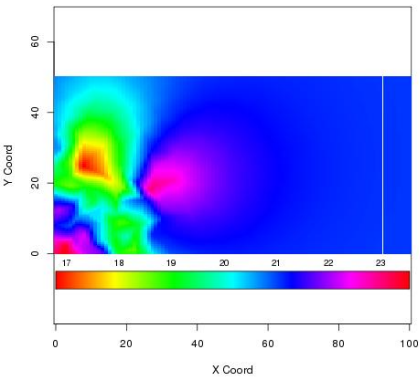

(c)

Fig. 3: Spatial field: (a) The true field; (b) The predicted field after 10 iterations with communication restrictions (c) The predicted field after 20 iterations with communication restrictions

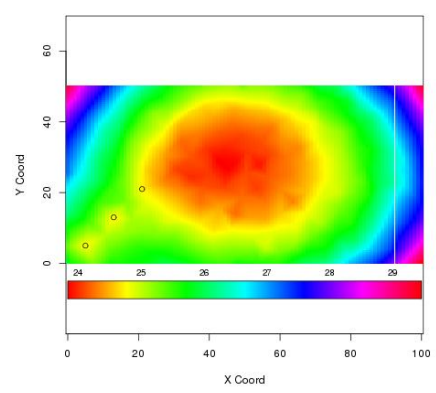

(a)

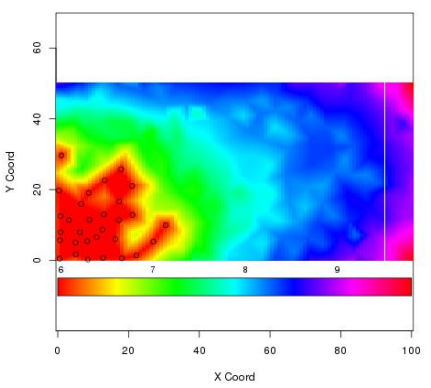

(b)

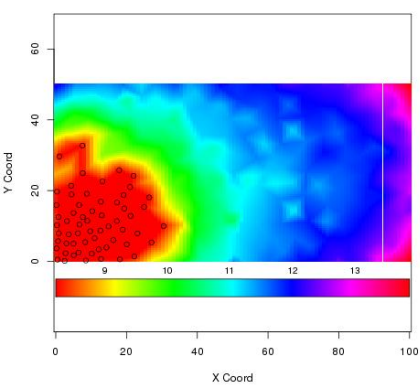

(c)

Fig. 4: Location of MRWSNs at time : (a) $t=1$ (Initial) (b) $t=10$, and (c) $t=20$.

the knowledge of the measurement noise $\sigma_{\varepsilon}^{2}=0.2$ and the system error $\sigma_{\text {sys }}^{2}=0.25$. The spatial field was represented by approximately 5000 vertices of triangles which were considered as spatially interested sites. In this scenario, percentage of non-zero elements in the sparse precision matrix is around $18 \%$.

The purpose of the first experiment is to characterise the nature of how a given number of MRWSNs capture information as they traverse through the spatial field subjected to the connectivity constraint. In this experiment we have initially used three MRWSNs positioned at $(5,5),(13,13)$ and $(21,21)$. Figures $3 \mathrm{~b}$ and $3 \mathrm{c}$ illustrate the predicted field after $t=10$ and $t=20$ steps respectively using three MRWSNs with connectivity constraint. We can clearly see that even though it is possible to use the GMRF effectively to drive the sensor nodes, as soon as the connectivity constraint is in place, MRWSNs are limited to a particular region. Afterwards, the RMSE gained by moving within this region is very limited.

The next experiment aims at demonstrating the proposed strategy. As described in Section (ref), when we detect only a minor variation in the RMSE of the predicted field between successive steps, we deem that as the 'best' a given set of MRWSNs deployed in the field can achieve as a team. At this point we send another MRWSN to join with others already in the field. The location of the new MRWSN is the most

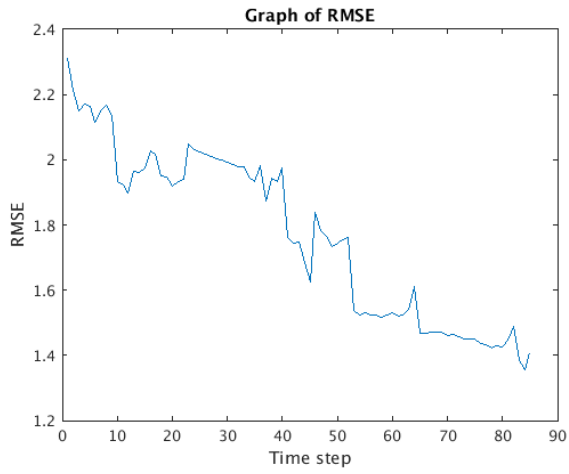

Fig. 6: Root Mean Squar Error

informative connected point relative to the furthest MRWSN in the field. This allows the area of coverage to grow out as shown in Fig. 5.

Fig.6 shows the resultant overall RMSE of the predicted field as new MRWSNs are joined to the existing team of MRWSNs.

\section{CONCLUSIONS}

In this paper, we have addressed the problem of modelling the physical phenomena under network connectivity constraints. The physical phenomena was modelled by a com- 


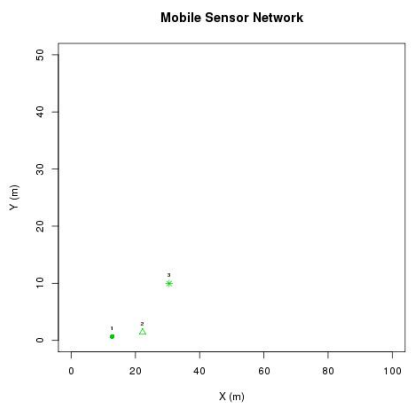

(a)

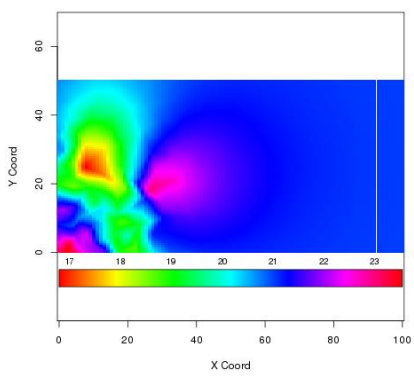

(e)

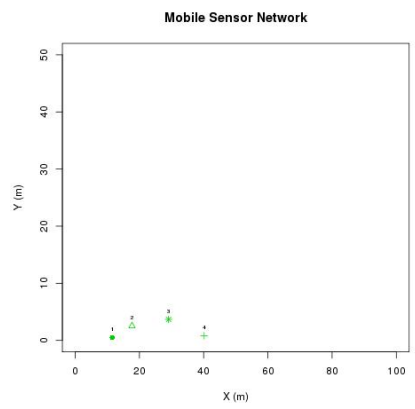

(b)

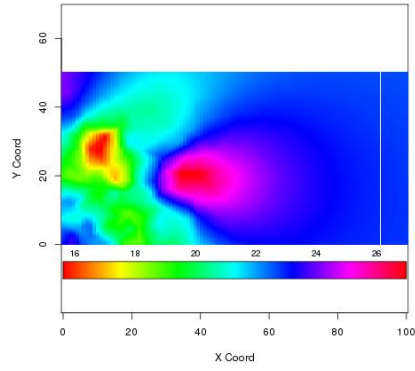

(f)

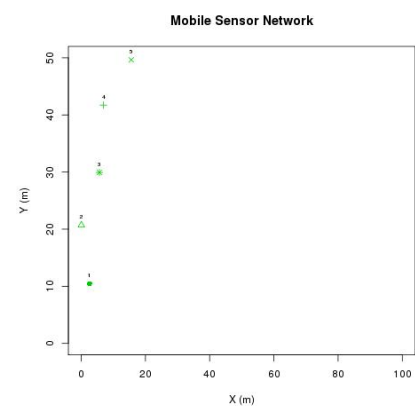

(c)

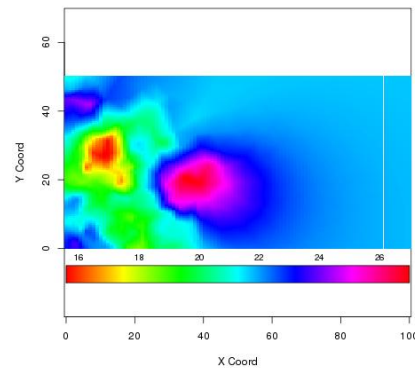

$(\mathrm{g})$

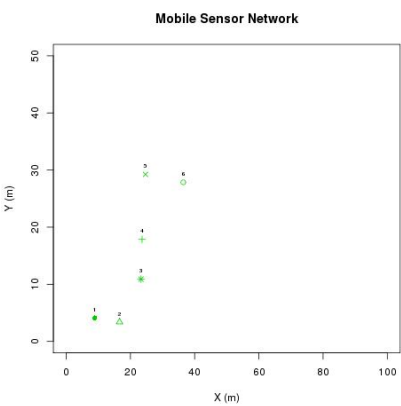

(d)

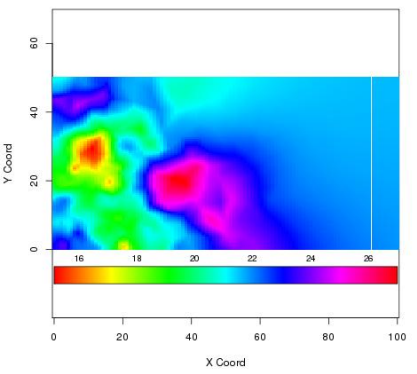

(h)

Fig. 5: Insertion of MRWSNs dynamically (a) initial deployment, $N=3$ (b) $N=4$ (c) $N=5$ (d) $N=6$, and the resultant predicted field under each case just before deploying the next MRWSN at time (e) $t=20$ (f) $t=39$ (g) $t=59$ (h) $t=79$

putationally efficient implementation of Gaussian processes, Gauss markov random fields. A published data set is used to validate the algorithm. The results clearly show that the MRWSN are striving to minimize the RMSE while strictly maintaining the connectivity. The connectivity is a very important aspect in the centralized network implementation. The results are appealing and demonstrate the demand for new MRWSN when it needs to further reduce the RMSE.

In the future, we expect to introduce more robotics and network constraints to make it more realistic. We also plan to implement the algorithms on real MRWSN.

\section{ACKNOWLEDGMENT}

This work is supported by the Centre for Autonomous System at University of Technology Sydney. Special thanks to Dr Van Linh Nguyen for helping with the code.

\section{REFERENCES}

[1] Z. Bojkovic and B. Bakmaz, "A survey on wireless sensor networks deployment," WSEAS Transactions on Communications, vol. 7, 2008.

[2] J. Yick, B. Mukherjee, and D. Ghosal, "Wireless sensor network survey," Computer Networks, vol. 52, 2008.

[3] J. H. Ryu, M. Irfan, , and A. Reyaz, "A review on sensor network issues and robotics," Journal of Sensors, 2015.

[4] A. Mainwaring, J. Polastre, R. Szewczyk, and D. Culler, "Wireless sensor networks for habitat monitoring," in Proc. of the 1st ACM international workshop on wireless sensor networks and applications, (New York, USA), 2002.
[5] M. Kirk, O. Royan, , and H. J. Glacsweb, "A sensor network for hostile environments," in Proceedings of the IEEE conference on sensing, communication and networking (SECON), (California, USA), pp. 8187, 2004.

[6] T. Fu, A. Ghosh, E. Johnson, and B. Krishnamachari, "Energy-efficient deployment strategies in structural health monitoring using wireless sensor networks," Struct Cont Health Monitor, vol. 20, pp. 971-986, 2013.

[7] T. Suzuki, R. Sugizaki, K. Kawabata, Y. Hada, and Y. Tobe, "Autonomous deployment and restoration of sensor network using mobile robots," International Journal on Advanced Robobotics Systems, vol. 7, pp. 105-114, 2010.

[8] S. Basagni, A. Carosi, and C. Petrioli, "Mobility in wireless sensor networks," Journal of Sensors, vol. 8, 2008.

[9] A. Howard, J. M. Matarie, and S. Gaurav, "Mobile sensor network deployment using potential fields: a distributed scalable solution to the area coverage problem," Distributed Autonomous Robot System, pp. 299-308, 2002.

[10] Y. Zou and K. Chakrabarty, "Sensor deployment and target localization based on virtual forces," in Proceedings of the IEEE International conference on computer communications (INFOCOMM), (San Francisco, USA), pp. 1293-1303, 2003.

[11] G. Tan, A. S. Jarvis, and A. M. Kermarrec, "Connectivity-guaranteed and obstacle-adaptive deployment schemes for mobile sensor networks," IEEE Transaction on Mobile Computing, pp. 836-848, 2009.

[12] G. Wang, G. Cao, and T. L. Porta, "Movement assisted sensor deployment," IEEE Transaction on Mobile Computing, pp. 640-652, 2006.

[13] N. Heo and K. P. Varshney, "A distributed self spreading algorithm for mobile wireless sensor networks," in Proceedings of the IEEE International Conference on Wireless communications and networking (WCNC), pp. 1597-1602, 2003.

[14] L. Nguyen, S. Kodagoda, R.Ranasinghe, and G. Dissanayake, "Information-driven adaptive sampling strategy for mobile robotic wireless sensor network," IEEE Transactions on Control Systems Technology, vol. 24, pp. 372-379, 2016. 
[15] P. J. Diggle and P. J. Ribeiro, Model-based geostatistics. New York, USA: Springer, 2007.

[16] C. E. Rasmussen and C. K. I. Williams, Gaussian Processes for Machine Learning. Cambridge, Massachusetts, London, England: The MIT Press, 2006.

[17] F. Lindgren, H. Rue, and J. Lindstrom, "An explicit link between gaussian fields and gaussian markov random fields: the stochastic partial differential equation approach," Journal of the Royal Statistical Society (Series B), vol. 73, pp. 423-498, 2011.

[18] A. Quarteroni and A. Valli, Numerical Approximation of Partial Differential Equations. Springer, 2008.

[19] C. M. Bishop, Pattern Recognition and Machine Learning. New York, USA: Springer, 2006.

[20] T. M. Cover and J. A. Thomas, Elements of Information Theory. John Wiley and Sons, Lnc,, 2006.

[21] R. Graham and J. Cortes, "Spatial statistics and distributed estimation by robotic sensor network," in Proceedings of the IEEE American Control Conference, pp. 2422-2427, 2010.

[22] Y. Xu and J. Choi, "Adaptive sampling for learning gaussian processes using mobile sensor networks," Sensors, vol. 11, pp. 3051-3066, 2011.

[23] Y. Xu, J. Choi, S. Dass, and R. Maiti, "Sequential bayesian prediction and adaptive sampling algorithms for mobile sensor networks," IEEE Transactions on Automatic Control, vol. 57, pp. 2078-2084, 2012.

[24] Y. Xu, J. Choi, S. Dass, and R. Maiti, "Efcient bayesian spatial prediction with mobile sensor networks using gaussian markov random elds," Automatica, vol. 49, pp. 3520-3530, 2013.

[25] M. Jadaliha, Y. Xu, and J. Choi, "Efficient spatial prediction using gaussian markov random fields under uncertain localization," in Proceedings of the ASME Dynamic Systems and Control Conference, 2012.

[26] C. Ko, J. Lee, and M. Queyranne, "An exact algorithm for maximum entropy sampling," Operations Research, vol. 43, pp. 684-691, 1995. 